\title{
Endoscope-Assisted Keyhole Technique for Hypertensive Cerebral Hemorrhage in Elderly Patients: A Randomized Controlled Study in 184 Patients
}

\author{
Yi FENG, Jianqing HE, Bin LIU, Likun YANG, Yuhai WANG \\ Anhui Medical University, School of Clinical Medicine (101-Hospital), Wuxi, China
}

\section{ABSTRACT}

\begin{abstract}
AIM: Hypertensive cerebral hemorrhage $(\mathrm{HCH})$ is a potentially life-threatening cerebrovascular disease with high mortality. In case of a massive hematoma, surgical drainage is a crucial treatment. The aim of the present study was to assess the efficacy of the endoscope-assisted keyhole technique in elderly patients with intracerebral hematoma who needed a flap craniotomy as traditional treatment.
\end{abstract}

MATERIAL and METHODS: One hundred-eighty-four elderly patients with $\mathrm{HCH}$, who had craniotomy indications after conservative treatment for 6-24 hours after onset, were randomly divided into two groups. In the craniotomy group, traditional hematoma drainage was performed. In the keyhole group, an endoscope-assisted keyhole technique was used. Anesthesia time, blood loss, hematoma drainage rate, and complications were compared. The clinical primary outcome was the six-month efficacy rate (defined by the activities of daily living (ADL) score).

RESULTS: Anesthesia time was longer in the craniotomy group ( $3.43 \pm 0.65$ vs. $1.53 \pm 0.52 \mathrm{~h}, \mathrm{P}<0.01)$, and blood losses were more important $(256 \pm 129$ vs. $96 \pm 39 \mathrm{ml} P<0.01)$. There was no difference in hematoma drainage rate between the two groups $(77.25 \pm 13.44$ vs. $83.52 \pm 27.51 \% \mathrm{P}>0.05)$. Complications, including tracheotomy $(\mathrm{P}<0.01)$, pulmonary infection $(\mathrm{P}<0.01)$ and hypoproteinemia $(\mathrm{P}<0.05)$ were more frequent in the craniotomy group. There was no difference in the occurrence of other complications, including revision surgery digestive tract ulcer and epilepsy. Proportion of patients with good prognosis (ADL I-III) was larger in the keyhole group $(\mathrm{P}<0.05)$.

CONCLUSION: In elderly $\mathrm{HCH}$ patients with an indication for hematoma drainage, better outcomes were achieved using an endoscope-assisted keyhole technique.

KEYWORDS: Hypertensive cerebral hemorrhage, Elderly, Endoscopy, Minimally invasive approach

\section{INTRODUCTION}

In the neurosurgical emergency ward, hypertensive cerebral hemorrhage $(\mathrm{HCH})$ is a common occurrence. Among cases of sudden stroke-related neurological deficits, spontaneous supratentorial intracerebral hemorrhage is common in Asians, has the highest morbidity and mortality, and accounts for $20 \%$ of all stroke cases. Risk factors for $\mathrm{HCH}$ increase dramatically with age (9).
The keyhole technique is a new minimally invasive surgical approach, and became a new trend in neurosurgery. Even if the indications for surgical drainage are still controversial, the criteria for the choice of a drainage technique are even less well established. Several groups developed minimally invasive clot removal techniques $(2,6,13,18)$.

In this present study, we focused on elderly patients with a surgical indication for hematoma drainage, and who were 
vulnerable to surgical risks, needing a shorter operative time, and a less invasive surgery comparing to younger patients. Endoscope-assisted small bone window hematoma drainage could provide a good solution in these patients.

\section{MATERIAL and METHODS}

\section{Study Design}

This was a prospective randomized clinical trial. The study was approved by the Ethical Committees of the 101 Hospital (China). The study was performed according to the Good Clinical Practice standards and to the Declaration of Helsinki. Since the patients included in this study were unable to provide an informed consent, written informed consent was obtained from the patients' legal guardians or healthy kindred.

\section{Patients}

Four hundred-seventy-four $\mathrm{HCH}$ patients admitted to our neurosurgery department between June 2006 and December 2013 were eligible. Inclusion criteria were: (1) aged 65 to 79 years; (2) presented with hypertension before or after onset, excluded from other causes of spontaneous intracerebral hemorrhage; (3) head computed tomography (CT) scan results showing subcortical, basal ganglia, internal capsule hemorrhage, accompanied or not by intraventricular hemorrhage; (4) patient received at least 6 hours of medical treatment, and bleeding was stopped according to CT; (5) Glasgow coma scale (GCS) score of 8-12; (6) strong indication for craniotomy (brainstem compression with midline shift $>1 \mathrm{~cm}$ ) and deterioration of consciousness (a fall in GCS score by 2 points or more). Exclusion criteria: (1) severe conscious disturbance or brain hernia; (2) thalamus or brainstem hemorrhage; (3) severe organ dysfunctions or clotting disorders; (4) hematoma volume larger than $60 \mathrm{ml}$ (inadequate indication for endoscopic operation (1, 15)); (5) other surgical contraindications.

One hundred-eighty-four patients were included and randomly divided into two groups using the random number generator in Microsoft Excel $\otimes$. Ninety-one patients were assigned to the craniotomy group (58 men and 33 women; mean age of 69.1 years). Ninety-three patients were assigned to the keyhole group (56 men and 37 women; mean age of 66.4 years) (Table I).

\section{Measurements}

The anesthesia time was recorded from the start of anesthesia to the end of operation. The hematoma volume was calculated by the Broderick's method (3). The hematoma drainage rate was calculated by dividing the preoperative hematoma volume by the postoperative hematoma volume. Complications occurrence, including tracheotomy, pulmonary infections, revision surgery, digestive tract ulcer and epilepsy, was compared between the two groups. Prognosis was assessed by the activities of daily living (ADL) score (10). I: no significant residual symptoms and signs, daily life completely normal; II: residual functional deficits, but able to take care of themselves every day; III: cannot take care of themselves on a daily basis, needing others' support; IV: cannot take care of themselves, but are aware; and V: vegetative state.

\section{Procedures}

In the keyhole group, all patients were treated by surgery under general anesthesia. The RUDOLF endoscope system was used (Rudolf Medical GmbH, Fridingen, Germany). First, the approximate range of hematoma was determined based on the CT results, and the location of the puncture path through the scalp was selected. A skin incision of about $5 \mathrm{~cm}$ was made before exposing the skull, and the size of the bone window was about $2.0 \times 2.0 \mathrm{~cm}$. Bone wax was used to stop bleeding, and the "cross" cut in the dura mater was made (if the hematoma volume was large, $250 \mathrm{ml}$ of $20 \%$ mannitol could be used for dehydration before cutting the dura mater). The cerebral cortex was coagulated, and paracentesis was performed to confirm hematoma. Using endoscopy, brain tissue was cut to form a tunnel with a diameter of about $1 \mathrm{~cm}$ following the puncture direction. Under direct vision, blood and clot were removed, and the endoscope was adjusted to reach the hematoma cavity. Most of the hematoma was removed under direct vision. Since the hematoma covering the bleeding source is often stiffly attached, the suction power was controlled, and this part of the clot was kept to avoid bleeding. At least $70 \%$ of total volume of hematoma was removed. To avoid brain tissue damage, the hematoma was removed under direct vision and hemostasis was completed by endoscopy. A silicone tube $(3.5 \mathrm{~mm}$ in outer diameter and 2.1 $\mathrm{mm}$ in inner diameter) was then inserted into the center of the hematoma cavity.

In the craniotomy group, all patients underwent general anesthesia and bone flap craniotomy decompression. According to the range of hematoma, a horseshoe-shaped incision was made in the frontotemporal area. The size of the bone window was approximately $6 \times 8 \mathrm{~cm}$. The length of incision in the surface of hematoma was $2-3 \mathrm{~cm}$. After hematoma removal, the dura mater was closed by tension suture. Bone flap decompression was performed depending on the extent of brain swelling.

Table I: Patients' Characteristics according to Surgical Treatment for $\mathrm{HCH}$

\begin{tabular}{lccc}
\hline & Craniotomy & Keyhole & P-value \\
\hline Gender (male/female) & $58 / 33$ & $56 / 37$ & 0.65 \\
\hline Age (years) & $69.10 \pm 10.26$ & $66.35 \pm 12.23$ & 0.10 \\
\hline Aspirin history (yes/no) & $21 / 70$ & $15 / 78$ & 0.27 \\
\hline Diabetes history (yes/no) & $11 / 80$ & $16 / 77$ & 0.41 \\
\hline
\end{tabular}




\section{Post-Operative Management}

Vital signs and vital organs functions were closely monitored. Airways were kept unobstructed and blood pressure was maintained stable. When using the keyhole technique, the bleeding source is always compressed by the residual hematoma rather than by electric coagulation; consequently, it is very important to control the blood pressure. Dexmedetomidine hydrochloride was commonly used to obtain a satisfying blood pressure in the first 2-3 days, which is the postoperative crucial period. In addition, blood pressure had to be maintained to a sufficient level to prevent any impairment of cerebral blood flow that could lead to low cerebral perfusion or cerebral infarction. All patients underwent CT examinations at the $1^{\text {st }}, 3^{\text {rd }}, 7^{\text {th }}$ and $14^{\text {th }}$ days post-surgery. All patients were followed-up for 6 months. Prognosis was assessed using the ADL score (10).

\section{Statistical Analysis}

STATA 7.0 (StataCorp, College Station, TX, USA) was used for data processing and analysis. The Student's t-test was used to compare anesthesia time, blood loss and hematoma drainage rate between the two groups. The $x^{2}$ test was used to compare the occurrence of each complication between the two groups. A P-value $<0.05$ was considered significant.

\section{RESULTS}

All patients achieved an improved GCS score after surgery. Postoperative CT scan showed a reduction in the midline shift. In the keyhole group, no case was converted to craniotomy. No postoperative brain hernia was observed. Figure 1A-D shows a patient with a large hematoma, in whom a successful drainage was performed by endoscopic keyhole surgery.

The anesthesia time and blood loss in each group are shown in Figure 2. Anesthesia time was longer in the craniotomy group $(3.43 \pm 0.65$ vs. $1.53 \pm 0.52 \mathrm{~h}, \mathrm{P}<0.01)$. The amount of blood loss was larger in the craniotomy group $(256 \pm 129$ vs. $96 \pm 39 \mathrm{ml}$, $\mathrm{P}<0.01)$. There was no difference in the hematoma drainage rate between the two groups $(77.25 \pm 13.44$ vs. $83.52 \pm 27.51 \%$, $\mathrm{P}>0.05)$. Table II shows that the complications and adverse events included tracheotomy, pulmonary infections, revision surgery, digestive tract ulcer, hypoproteinemia and epilepsy. Prognosis evaluation for the two groups according to the ADL score 6 months after surgery is shown in Table III.

\section{DISCUSSION}

In the past, removing the hematoma by routine craniotomy was the main surgical treatment for $\mathrm{HCH}$. However, this approach had the main disadvantage of an important surgical
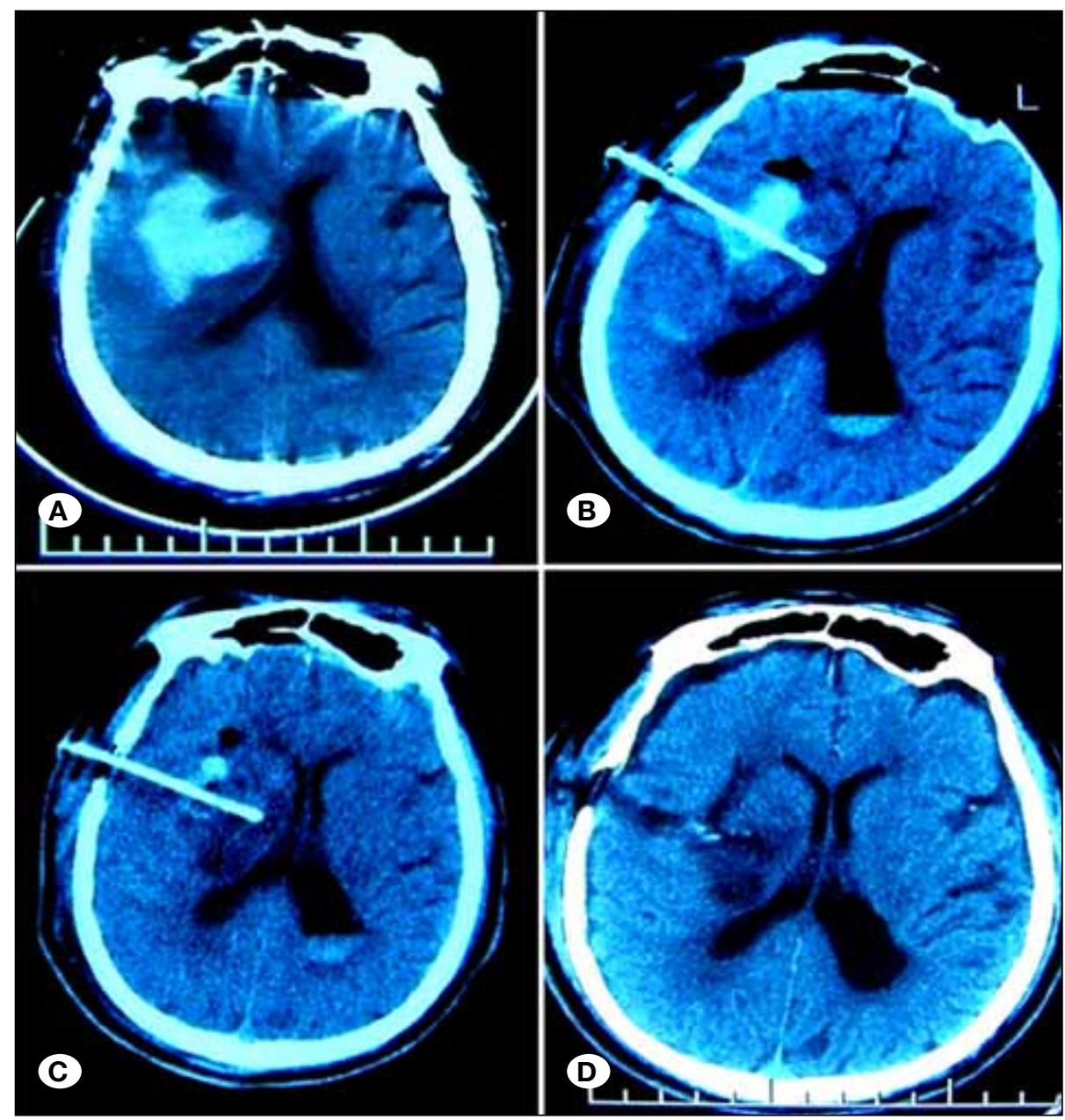

Figure 1: A patient (male, 71 years old) was admitted 7 hours after $\mathrm{HCH}$. A) Preoperative $\mathrm{CT}$, the hematoma in the right basal ganglia was about $40 \mathrm{ml}$. B) CT scan at the $1^{\text {st }}$ postoperative day. Most of the hematoma was removed. The volume of the residual hematoma was about $15 \mathrm{ml}$. C) CT image at the $3^{\text {rd }}$ postoperative day, before removing the drainage tube.

D) CT image at the $7^{\text {th }}$ postoperative day. 


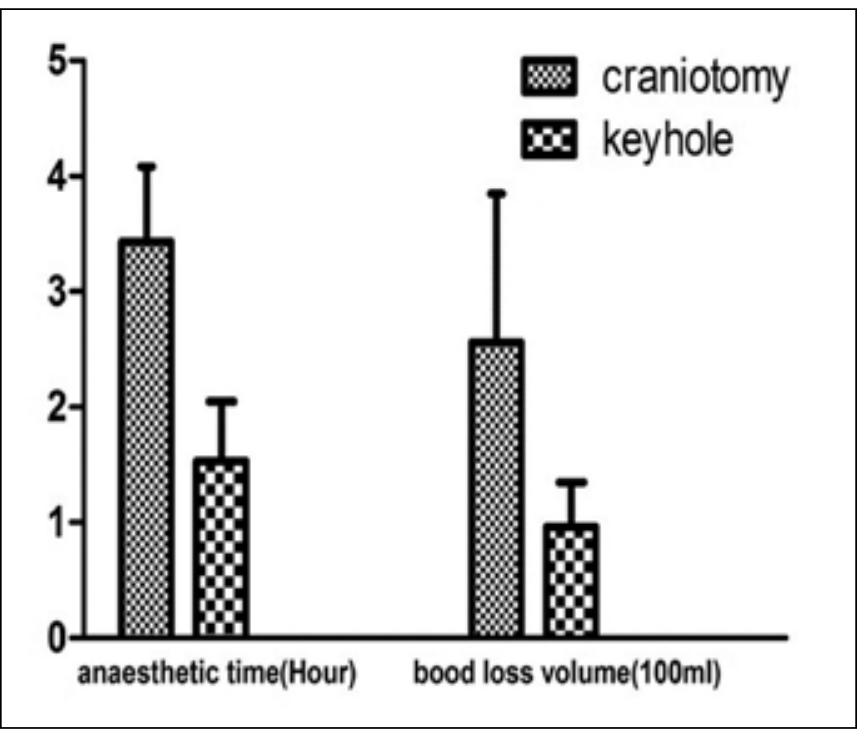

Figure 2: Comparison of the anesthesia time and blood loss volume between the two groups.

trauma, long general anesthesia, further bleeding, and more impact on brain tissues. Nowadays, surgical treatment of $\mathrm{HCH}$ includes conventional surgical craniotomy, keyhole craniotomy, simple trepanation and drainage, and stereotactic hematoma aspiration. Whatever the surgical technique, the approach used should not add to the existing mass effect via postoperative edema, and should not lead to further bleeding. The endoscope-assisted keyhole technique has been used in brain surgery for a number of indications, such as for aneurysms (7) and tumors (5).

Elderly patients with an atrophic brain have a lower intracranial pressure (ICP) compared with younger patients with the same hematoma size, and they have more time to wait until the bleeding stop. Most $\mathrm{HCH}$ have no growth in hematoma volume after 6 hours following onset (4). Although the bleeding is stopped, the upcoming edema and low consciousness status remain threatening. Traditional craniotomy may be associated with a longer anesthesia, significant blood loss and trauma in elderly patients, which may easily induce complications like hypoproteinemia and pulmonary infection. As shown in an epidemiological study of 266 cases of intracerebral hemorrhage from Hangzhou (China) (9), pulmonary infection accounts for the most frequent complication in these patients. Deaths in the first month, but beyond the first few days following $\mathrm{HCH}$ onset, is usually the result of complications such as pulmonary infection, intracranial infection, respiratory failure, and multiple organ failure (9). In elderly patients with $\mathrm{HCH}$, it is essential to select a minimally invasive treatment leading to less brain retraction and brain tissue injury, with shorter anesthesia time and less blood loss. The keyhole technique dramatically reduces the size of craniotomy with a smaller dura mater opening, less brain exposure, and less retraction of brain tissue. The incidence of pulmonary infection is associated with anesthesia time, state of nutrition, and state of consciousness. Consequently, the decreased anesthesia time, blood loss and hypoproteinemia associated with the keyhole technique contributed to less pulmonary infections being observed in the present study. In addition, we found in our clinical practice that brain over-harassment caused by an unskilled surgeon may cause an increased brain edema (even if no bleeding is found), leading to a late consciousness recovery. Fewer complications secondary to changes in cerebral circulation (especially epilepsy) were found using the keyhole technique (8), which may possibly be due to smaller cortex exposure and to the avoidance of direct brain compression due to a change in the patient's position after decompression craniotomy.

Other than the benefit of a smaller trauma, the keyhole technique uses endoscopy, which has a number of advantages, including high lighting intensity, flexibility, multi-angle observation and "observe around the corner" capability, which might make up for the insufficiency of direct vision. Hematoma removal with endoscope-assisted surgery under direct vision could decrease brain tissue stretch. At the end of surgery, surgeons are able to ensure that there is perfect hemostasis. Using endoscopy, there may be limitations in the flexibility of the instrument and in controlling bleeding from major vessels. Under such circumstances, the surgeon may have to switch to open microsurgery.

In the present study, we found that the keyhole technique was reliable, that there was no difference in the drainage rate, that there were fewer complications like pulmonary infection, hypoproteinemia, and epilepsy, and that a smaller number of tracheotomies were performed. There was no difference in adverse events incidence such as revision surgery and digestive tract ulcer between the two groups. Qiu et al. (19) treated 25 hypertensive intracranial hematomas patients (mean age of $62 \pm 8$ years) using endoscope-assisted keyhole drainage, and they reported a better hematoma drainage rate and better clinical outcomes compared with traditional drainage.

The main challenge for this study was that when a keyhole technique was selected, surgeons could have difficulties in finding the most appropriate cortical cutting site. Indeed, some functional areas which can be preserved using traditional craniotomy may be damaged due to the small cortex exposure, and a worse neurological impact may happen especially in cases of dominant hemisphere and in cases of deep hematoma. Nevertheless, we observed better outcomes by using the keyhole technique, which may be due to the fact that the cortical cutting site does not matter since the nerve conduction beam was already been damaged. More investigation must be performed on that subject.

Minimally invasive surgical clot removal techniques are still controversial (14), and have been reported to increase clot removal and to decrease mortality in certain patients, but no improved outcomes wee reported $(2,6,18)$. Hematoma drainage by open microsurgery, endoscope-assisted surgery or image-guided drainage with rTPA are being studied in clinical trials (such as in the MISTIE trial), but the results are not yet available. Drainage of hematoma in selected cases may benefit the patient, but it has not been proved in controlled studies. Mendelow et al. (12) performed a prospective ran- 
Feng Y. et al: Keyhole Technique for Hypertensive Cerebral Hemorrhage

Table II: Occurrence of Complications according to the Type of Surgery

\begin{tabular}{lccc}
\hline Complication & Craniotomy $\mathbf{( n = 9 1 )}$ & Keyhole $(\mathbf{n}=\mathbf{9 3})$ & P-value \\
\hline Tracheotomy & 29 & 13 & 0.005 \\
\hline Pulmonary infection & 38 & 14 & 0.000 \\
\hline Revision surgery & 3 & 6 & 0.49 \\
\hline Digestive tract ulcer & 3 & 2 & 0.68 \\
\hline Hypoproteinemia & 22 & 9 & 0.01 \\
\hline Epilepsy & 15 & 6 & 0.04 \\
\hline
\end{tabular}

$X^{2}$ tests.

Table III: Prognosis of Patients according to the Type of Surgery and to the ADL Score

\begin{tabular}{lccccccc}
\hline \multirow{2}{*}{ Group } & $\mathbf{n}$ & I & II & III & IV & V & VI (death) \\
\cline { 3 - 6 } & 91 & 4 & 13 & 22 & 20 & 24 & 8 \\
\hline Craniotomy & & & Good recovery & & & & \\
\hline Total & 93 & 8 & 18 & 34 & 11 & 16 & 6 \\
\hline Keyhole & & & 60 & & & & \\
\hline Total & & & & & & & \\
\hline
\end{tabular}

Grades I-III are regarded as good recovery. Rates were compared using $X^{2}$ tests.

domized trial (the STICH trial) to compare early surgery with initial conservative treatment for patients with intracerebral hemorrhage; they observed a small benefit from surgeries for hematomas located near the cortical surface. Urokinase, rTPA or other agents dissolving clots were not used in this present study, because most of the clot had been cleaned out, and the residual clot could be drained using drainage tubes, which was kept for 2 weeks without infection in several cases. Clot dissolving itself may add the risk of intracranial infection and chemical stimulus.

Is surgical treatment better than conservative management? There is still no strong evidence. Cases selected in this study were patients with strong indications of surgical treatment who were not supposed to survive without surgical drainage, according to our clinical experience. Niizuma et al. (17) investigated thalamic hematoma patients, including 75 stereotactic aspirated cases and 70 non-aspirated cases: results suggest that aspiration can improve therapeutic results. The trial completed by Auer et al. (2) in 1989 studied patients with a hemorrhage $>10 \mathrm{~cm}^{3}$ in volume, and showed a $28 \%$ lower mortality in the endoscopic surgery group compared with the medical treatment group after 6 months of follow-up. However, surgical benefit was mainly limited to patients with lobar hematomas and to patients $<60$ years old. These results contradict ours, which may be due to the fact their patients had a smaller hematoma volume and required less aspiration. The $\mathrm{ECMOH}$ trial (20) was a randomized controlled trial designed to evaluate if endoscopic surgery was better than conservative treatment for patients with moderate-volume hematomas in the basal ganglia, but the results have not been published yet.

Hematoma drainage was reported earlier (11), and may save the life of many patients. However, this technique could not effectively lower the local pressure. Indeed, it was reported that the pressure in the hematoma cavity was higher than the intracranial pressure (ICP) of $\mathrm{HCH}$ patients. Brain tissue damage around the hematoma was more serious than the whole brain damage caused by high ICP. The longer the hematoma compression time was, the more serious the brain tissue damage around the hematoma (16). Another disadvantage of burr-hole compared with the keyhole technique is that burr-hole cannot be performed under eye vision.

CT scans allows choosing a very precise approach to a specific lesion before operation. In Matsumoto's study (11), threeor two-dimensional CT images were taken to determine the coordinates of the target point, which was the center of the hematoma. However, we recommend that the target point should be behind the center by $1-2 \mathrm{~cm}$, with the aim of reducing the risk of lenticulostriate artery (also known as artery of cerebral hemorrhage). However, the hematoma is prone to drop backwards during suction, which may also provide a better access to the hematoma part near the brain stem.

In conclusion, we found that elderly $\mathrm{HCH}$ patients who had strong indications of clot removal had better outcomes if an endoscope-assisted keyhole technique was used. Indeed, the endoscope-assisted keyhole technique effectively prevented the occurrence of brain hernia without significant harm done to the patients. This technique demonstrated sufficient de- 
compression, smaller incision, shorter operation time, smaller bleeding volume, lesser complications, and improved longterm efficacy and prognosis. Therefore it is necessary to reevaluate the surgery indications for hypertensive hematoma in elderly patients.

\section{REFERENCES}

1. Agmazov MK, Bersnev VP, Ivanova NE, Pavlov OA, Nikitin Al, Akhtamov DA, Arzikulov TN: Minimally invasive surgery of patients with hypertensive intracerebral bleedings. Vestn Khir Im I I Grek 168: 78-82, 2009

2. Auer LM, Deinsberger W, Niederkorn K, Gell G, Kleinert R, Schneider G, Holzer P, Bone G, Mokry M, Korner E: Endoscopic surgery versus medical treatment for spontaneous intracerebral hematoma: A randomized study. J Neurosurg 70: 530-535, 1989

3. Broderick JP, Brott TG, Tomsick T, Barsan W, Spilker J: Ultra-early evaluation of intracerebral hemorrhage. J Neurosurg 72: 195-199, 1990

4. Brott T, Broderick J, Kothari R, Barsan W, Tomsick T, Sauerbeck L, Spilker J, Duldner J, Khoury J: Early hemorrhage growth in patients with intracerebral hemorrhage. Stroke 28: 1-5, 1997

5. Charalampaki P, Filippi R, Welschehold S, Conrad J, Perneczky A: Tumors of the lateral and third ventricle: removal under endoscope-assisted keyhole conditions. Neurosurgery 62: 10491058, 2008

6. Cho DY, Chen CC, Chang CS, Lee WY, Tso M: Endoscopic surgery for spontaneous basal ganglia hemorrhage: Comparing endoscopic surgery, stereotactic aspiration, and craniotomy in noncomatose patients. Surg Neurol 65: 547-555; discussion 555556, 2006

7. Fries G, Perneczky A: Endoscope-assisted keyhole surgery for aneurysms of the anterior circulation and the basilar apex. Operative Techniques in Neurosurgery 3: 216-230, 2000

8. Hirasawa $\mathrm{K}$, Kasuya $\mathrm{H}$, Hori T: Change in circulating blood volume following craniotomy. J Neurosurg 93: 581-585, 2000

9. Hu YZ, Wang JW, Luo BY: Epidemiological and clinical characteristics of 266 cases of intracerebral hemorrhage in Hangzhou, China. J Zhejiang Univ Sci B 14: 496-504, 2013

10. Kwiatkowski TG, Libman RB, Frankel M, Tilley BC, Morgenstern LB, LuM, Broderick J P, Lewandowski CA, Marler JR, Levine SR, Brott T: Effects of tissue plasminogen activator for acute ischemic stroke at one year. National Institute of Neurological Disorders and Stroke Recombinant Tissue Plasminogen Activator Stroke Study Group. N Engl J Med 340: 1781-1787, 1999
11. Matsumoto $\mathrm{K}$, Hondo $\mathrm{H}$ : CT-guided stereotaxic evacuation of hypertensive intracerebral hematomas. J Neurosurg 61: 440-448, 1984

12. Mendelow AD, Gregson BA, Fernandes HM, Murray GD, Teasdale GM, Hope DT, Karimi A, Shaw MD, Barer DH: Early surgery versus initial conservative treatment in patients with spontaneous supratentorial intracerebral haematomas in the International Surgical Trial in Intracerebral Haemorrhage (STICH): A randomised trial. Lancet 365: 387-397, 2005

13. Morgan T, Zuccarello M, Narayan R, Keyl P, Lane K, Hanley D: Preliminary findings of the minimally-invasive surgery plus rtPA for intracerebral hemorrhage evacuation (MISTIE) clinical trial. Acta Neurochir Suppl 105: 147-151, 2008

14. Morgenstern LB, Hemphill JC 3rd, Anderson C, Becker K, Broderick JP, Connolly ES Jr, Greenberg SM, Huang JN, MacDonald RL, Messe SR, Mitchell PH, Selim M, Tamargo RJ: Guidelines for the management of spontaneous intracerebral hemorrhage: A guideline for healthcare professionals from the American Heart Association/American Stroke Association. Stroke 41: 2108-2129, 2010

15. Nakano T, Ohkuma H, Ebina K, Suzuki S: Neuroendoscopic surgery for intracerebral haemorrhage--comparison with traditional therapies. Minim Invasive Neurosurg 46: 278-283, 2003

16. Niizuma $H$, Suzuki J: Stereotactic aspiration of putaminal hemorrhage using a double track aspiration technique. Neurosurgery 22: 432-436, 1988

17. Niizuma H, Yonemitsu T, Jokura $H$, Nakasato N, Suzuki J, Yoshimoto T: Stereotactic aspiration of thalamic hematoma. Overall results of 75 aspirated and 70 nonaspirated cases. Stereotact Funct Neurosurg 54-55: 438-444, 1990

18. Nishihara T, Morita A, Teraoka A, Kirino T: Endoscopy-guided removal of spontaneous intracerebral hemorrhage: Comparison with computer tomography-guided stereotactic evacuation. Childs Nerv Syst 23: 677-683, 2007

19. Qiu Y, Lin Y, Tian X, Luo Q: Hypertensive intracranial hematomas: Endoscopic-assisted keyhole evacuation and application of patent viewing dissector. Chin Med J (Engl) 116: 195-199, 2003

20. Zan X, Li H, Liu W, Fang Y, Ma J, Lan Z, LiX, Liu X, You C: Endoscopic surgery versus conservative treatment for the moderate-volume hematoma in spontaneous basal ganglia hemorrhage $(\mathrm{ECMOH})$ : Study protocol for a randomized controlled trial. BMC Neurol 12: 34, 2012 